\title{
Diagnosis methods for mechatronic systems
}

\author{
Syrine Derbel ${ }^{1,2}$, Nabih Feki ${ }^{2}$, Florentina Nicolau ${ }^{1}$, Jean Pierre Barbot ${ }^{1}$, \\ Mohamed Slim Abbes ${ }^{2}$, and Mohamed Haddar ${ }^{2}$ \\ 1 Quartz Laboratory / ENSEA, Cergy, France 95014, \\ syrina.derbel@hotmail.fr, \\ 2 LA2MP Laboratory / ENIS, Sfax, Tunisia 3038
}

\begin{abstract}
Mechatronic systems are widely used in many industrial applications for their capacity to improve the production performances. However, due to the complexity of the mechatronic systems, several faults that disrupt the system operating, may occur and specially, the dynamic defects (e.g., electrical, mechanical, design defects $\cdots$ ) and the sensors faults (e.g., bias or gain defects). In this paper, classification of some diagnosis methods is presented and a new diagnosis technique, called sparse recovery, is presented. Academic example is developed in order to illustrate the good performance of the proposed method.
\end{abstract}

Keywords: diagnosis methods, sparse recovery, mechatronic systems.

\section{Introduction}

\section{Problem statement}

Generally, mechatronic systems are complex systems which are composed of at least two interconnected subsystems. Thus, they can be presented under a non linear state-space representation:

$$
\left\{\begin{array}{l}
\dot{x}=f(x, t)+g(x, t) u \\
y=h(x)
\end{array}\right.
$$

where $x \in \mathbb{R}^{n}$ is the state vector of the system, $u \in \mathbb{R}^{p}$ is the input vector and $y \in \mathbb{R}^{m}$ is the output vector, $f, g$ and $h$ are vector field assuming sufficiently smooth. In mechatronic application, the state vector $x$ must include all state (variables) obtained from the subsystems modeling of the global system. For instance, a mechanical subsystems can be modeled considering, as a state vector, the degree of freedom of the system (Derbel et al. 2019). Concerning an electrical application, the state vector can be represented by the currents, fluxes or rotational speed variables (Feki et al. 2010). In thermodynamic applications, the state space vector can be represented by the heat flow density or the environmental temperature (Galant et al. 2016).

Each mechatronic systems can be affected by several defects which are considered and modeled as following:

$$
\left\{\begin{array}{l}
\dot{x}=f(x, t)+g(x, t) u+D_{1}(x, t) w(t) \\
y=h(x)+D_{2}(t) w(t)
\end{array}\right.
$$


where $D_{1}(x, t), D_{2}(t)$ are the fault matrices related to, respectively, the operating systems and the sensor faults, $w(t)$ represents the possible fault vector. Diagnostic of these faults is a difficult issue for industries and any fault modeled in the (2) needs to be successfully and efficiently detected, localized and identified using a limited set of sensor signals available.

\section{Diagnosis methods}

The classification of the diagnostic methods is not unique and depends on the specific branch that researchers are focused (see, i.e.,(Hwang et al. 2010),(Mouzakitis 2013) and (Yu et al. 2014)). In this paper, the proposed classification is illustrated in Fig. 1 and is classified into two areas: the hardware redundancy and the analytical redundancy. The basic concept of the hardware redundancy is to use multiple hardware, as the sensors measurements (two or three sensors), in order to analysis the same information and to diagnosis faults. However, the analytical redundancy requires the knowledge of specific information given from a mathematical model, a signals (frequency) information or from a historic system data. Each category is discussed briefly in the following subsections.

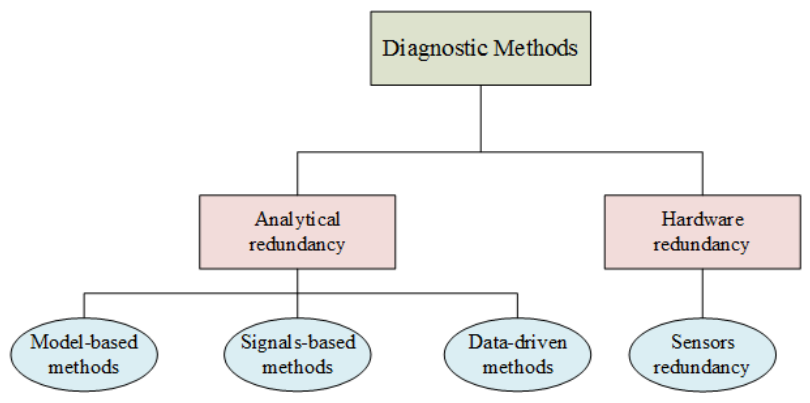

Fig. 1: Classification of diagnostic methods

\subsection{Hardware redundancy}

The key idea of the hardware redundancy is to use several materials in order to measure the same signals. The hardware redundancy deals with the comparison of duplicative signals generated by more than one sensor using algebraic relation between different system variables. The advantage of this method appears in its reliability and its simplicity. On the other hand, this diagnosis method presents a major setback which is the expensive cost of maintenance due to the extra equipment. An additional space is required to place the extra equipment that limits also the use of this method. 


\subsection{Analytical redundancy}

The analytical redundancy is mainly based on specific information given by local or global modeling of the system. In this article, the analytical redundancy is divided into three categories: model-based methods, signals-based methods and data driven methods (see Fig. 1).

Model-based methods Model-based methods are most effective methods compared to the hardware redundancy because it does not require additional hardware. Model-based methods are based on a mathematical model obtained by

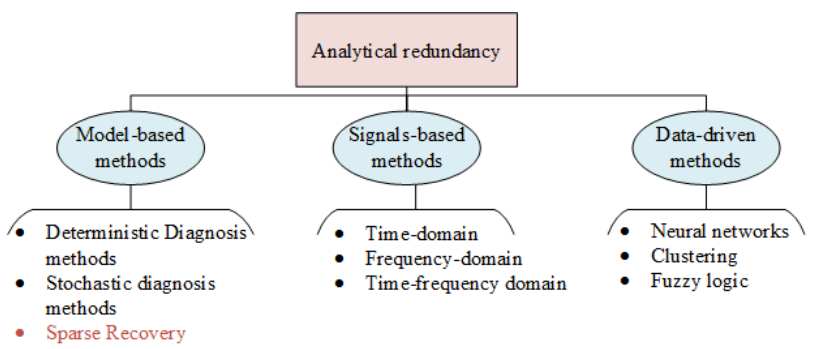

Fig. 2: Classification of diagnostic methods

developing some physical and fundamental principles. Generally, model-based methods allows to compare the available measurements of the practical systems and the model predicted outputs. In this section, the principle of some modelbased methods (see, Fig.2) is detailed and divided, with respect to the type of the model, into three categories: deterministic diagnostic methods, stochastic diagnostic methods. Sparse recovery method belongs to model-based diagnosis methods since it requires a mathematical model that describes the system behavior.

\section{- Deterministic diagnosis methods}

The deterministic diagnosis methods are based on a deterministic modeling of a system under consideration. They can be presented in two main areas: parity space and diagnostic based on observers.

Parity space: The principle of the parity space is to create residual which is, generally, the difference between the model and the system outputs. With the help of the residual information, the consistency between this difference is checked. Without faults, the residual generated by the party space technique is equal to zero. However, it is non-zero if modeling errors, noises or faults occur in the system. The parity space method can be used to either the time-domain statespace model or frequency-domain model. This diagnostic method is applied for 
many complex model, for example, to estimate faults for a quadrotor unmanned aerial vehicle (Han et al. 2018), to detect fault for electro-mechanical brake systems (Hwang \& Huh 2015), ect,.... However, in some dynamical system, due to the lack of the algebraic relations between different system variables, the parity space method becomes unable to diagnosis some specific faults.

Diagnostic based on observers: Diagnostic observers play a major role in the deterministic diagnosis methods and,generally, they are based on a space-state representation of the system. These diagnosis methods can be used to detect, or isolate, or identify the fault. An important issue of the diagnosis methods based on observers is the choice of the observer gain that must make the residual signals sensible to faults and insensible to disturbances (modeling errors, noises and process disturbances). Precisely, the gain observer is chosen in order to minimize the ratio between the residual term linked to the disturbance and the residual term related to the faults. In order to solve this optimization problem ((Gao, Cecati \& Ding 2015)), the common design approach is the eigenstructure assignment method. It gives a design of the gain observer by using the left and right eigenstructure methods and aims to cancel directly the disturbance (for more details, see (Xu \& Tseng 2007)). Recently, diagnostic observers are used in many applications in order to diagnosis damage (see, e.g., (Odofin et al. 2016), (Sen \& Bhattacharya 2017) and (Odofin et al. 2018)). Residual generation using unknown input observer is a important diagnostic method and applied specially to isolate the fault. The key idea of the unknown input observer is to give state estimation of the system by decoupling the unknown input related to the disturbances (see, for more details (Edwards et al. 2000) and for applications (e.g.,(Alhelou et al. 2018), (Gao, Liu \& Chen 2015)). Concerning the fault identification, many types of observers are developed (for instance, adaptive observers (Rodrigues et al. 2015), sliding mode observers (Barbot et al. 1996), proportional integral observer (Youssef et al. 2017), ect,...). The concept of these observers is that, in the modeling system, the considered fault are implemented as an additional state and thus, the global extended state vector will be estimated including the fault and original signals. Recently, the combination of different type of observers is an important topic of researchers in order to diagnosis several faults (e.g., citepob10, (Chen et al. 2016), (Rahme \& Meskin 2015)).

\section{- Stochastic diagnosis methods}

Stochastic diagnosis methods are a model-based methods in which the model process some inherent randomness.

Kalman filter: The common stochastic diagnostic methods is the residual generation using Kalman filter. The defects are diagnosed by statistical testing on whiteness, co-variance and mean of the residuals. Several statistical tools are developed in order to test the presence of faults such as maximum likelihood method, generalized likelihood ration, ect,... (see, (Gao, Cecati \& Ding 2015)), for more information). Kalman filer has been modified in many research works 
in order to improve the accurately of the stochastic approximation (unscented Kalman filter (Sepasi \& Sassani 2010), to consider the non linear system (extended Kalman filter (Foo et al. 2013)) or to adjust the noise (adaptive Kalman filter (Kim et al. 2009)).

Parameter estimation: Parameter estimation is another stochastic diagnostic methods which consider that the presence of fault lead to a change in the system parameter (for example, space matrix in the space-state representation). The basic structure in healthy state is known and compared to the estimated parameters methods in order to obtain fault information (see, for example, (Bachir et al. 2006)).

\section{- Sparse recovery method}

Sparse recovery method is a new technique used to detect, isolate and identify the faults in dynamical system. It is a diagnostic method based on system modeling specially on the state-space representation. The unknown inputs of the system (faults, disturbances, attacks) are reconstructed under the condition that only specific number of the inputs are detected of a long list of possible inputs. Some other conditions must be verified in order to diagnosis faults. Sparse recovery method is widely applied in image processing and recently, this method becomes a diagnostic methods to diagnosis dynamical system. For instance, cyber-attack reconstruction via sparse recovery algorithm for electrical power networks application is developed in (Nateghi et al. 2018). The problem of sparse recovery is an optimization program that minimizes a cost function constructed by leveraging the observation error term and the sparsity inducing term:

$$
w^{*}=\min _{w \in \mathbf{R}^{p}}\left\{\frac{1}{2}\|M-H w(t)\|_{2}^{2}+\lambda\|w(t)\|_{1}\right\} .
$$

with $w$ is the estimated fault vector, $M$ is the observed and available measurement and $H$ is a sensing matrix determined form the fault modeling.

Model-based methods are an important diagnostic methods that need to have a large knowledge of system modeling (the physical laws, behavior system, ect,.... Moreover, the frequency characteristics and behavior of the system are also an essential information to generate a perfect system modeling. This need led to the other diagnostic methods that need the frequency knowledge of the system.

Signals-based methods Signal-based methods are depend on measured signals which contain the fault characteristics. In this section, signal based methods are divided into three areas: time-domain methods, frequency-domain methods and time-frequency methods.

Time-domain methods: Time-domain methods are the basic diagnostic methods based on extraction of time-domain characteristics of the measured signals. The main advantage in using the time-analysis is the period of time that can be associated with some form of fauls. The common time-domain methods are: 
root-mean-square, crest factor, absolute value, kutosis, ect,.... These diagnostic methods are used in widely industrial application in order to detect faults in different systems (see for example, (Tian et al. 2015),(Hong \& Dhupia 2014), and (Jan et al. 2017)). The early methods possess some limitations such as the difficulty faults detection in a noisy environment and in specific case the localization and the identification of the faults are not always possible.

Frequency-domain methods: Frequency-domain methods is a method to detect abnormality or defect by using the spectrum analysis tools. The Fast Fourier Transform FFT is the most applied method in frequency analysis technique. It aims to extract the fault indication from the vibration measured on the machine and to analyze the frequency characteristics and the amplitude of the defect. Though the FFT has the ability of frequency analysis for the stationary signals, it cannot illustrate the change in the frequency spectra for a non-stationary signal (Gong \& Qiao 2015).Therefore, FFT cannot reveal the hidden fault information in a non-stationary signal. In specific cases, the spectrum is not always readable and clear and not contain information if changes occur on the transmission path. Thus, the cepstral analysis is used and deal with the identification of the damage in the situation of the combined effects of harmonics. The spectral analysis is used for many industrial applications such as diagnostic and analysis of the behavior of electric motors and electronic systems (Sonavane \& Jadhav 2015), detection to tooth crack from electrical measurement (Feki et al. 2013), monitoring of the wind behavior (Gayatri \& Sekhar 2018).

Time-frequency methods: Time-frequency methods are used specially to analysis a non-stationary signals. They aims to identify the frequency characteristic of signals and to extract their time variant features which can be an effective tools for diagnostic. The common time-frequency methods are the short-time-Fourier transform, wavelet transform, Hilbert transform. For instance, In (Cao et al. 2016),(Burriel-Valencia et al. 2017), the time-frequency methods are applied in order to diagnosis faults in dynamic systems working in transient state.

The frequency knowledge is an important tool for the diagnostic specially in mechatronic system. This knowledge is one of the required tools that allows to analyze the system behavior if the system modeling is not available. So, the data-driven methods describe process behavior based on available empirical or experiential information from sensors systems and/or human operator.

Data-driven methods Data-driven methods are the diagnostic methods that not require a mathematical model of the physics. The key idea of data-driven methods is to generate a model of the process, which related measured inputs to measured outputs and then use this model against the real process to create residual. Fuzzy logic, neural networks, and clustering are the common diagnostic methods of the data driven methods.

Fuzzy logic: Fuzzy logic is a mathematical method that solve different simulated problems based on many inputs and output. Fuzzy logic is able to give results 
in the form of recommendation for a specific interval of output state using a knowledge base including data and role data (Adhikari et al. 2016).

Neural networks Neural networks are intelligent systems inspired from biological neural networks and are mostly suited for faults diagnosis in non-linear systems. Neural networks give a mapping between the inputs and the outputs of the system and form an associate memory that recovers the appropriate output when presented with an unseen input. For instance, this diagnostic method is applied (Er-raoudi et al. 2016) in order to detect gear fault.

\section{Examples}

\section{Conclusions}

In this paper, classification of different diagnostic methods is presented. 


\section{Bibliography}

Adhikari, S., Sinha, N. \& Dorendrajit, T. (2016), 'Fuzzy logic based on-line fault detection and classification in transmission line', SpringerPlus 5(1), 1002.

Alhelou, H. H., Golshan, M. H. \& Askari-Marnani, J. (2018), 'Robust sensor fault detection and isolation scheme for interconnected smart power systems in presence of rer and evs using unknown input observer', International Journal of Electrical Power \& Energy Systems 99, 682-694.

Bachir, S., Tnani, S., Trigeassou, J.-C. \& Champenois, G. (2006), 'Diagnosis by parameter estimation of stator and rotor faults occurring in induction machines', IEEE Transactions on Industrial Electronics 53(3), 963-973.

Barbot, J.-P., Boukhobza, T. \& Djemai, M. (1996), Sliding mode observer for triangular input form, in 'Proceedings of 35th IEEE Conference on Decision and Control', Vol. 2, IEEE, pp. 1489-1490.

Burriel-Valencia, J., Puche-Panadero, R., Martinez-Roman, J., Sapena-Bano, A. \& Pineda-Sanchez, M. (2017), 'Short-frequency fourier transform for fault diagnosis of induction machines working in transient regime', IEEE Transactions on Instrumentation and Measurement 66(3), 432-440.

Cao, H., Fan, F., Zhou, K. \& He, Z. (2016), 'Wheel-bearing fault diagnosis of trains using empirical wavelet transform', Measurement 82, 439-449.

Chen, F., Zhang, K., Jiang, B. \& Wen, C. (2016), 'Adaptive sliding mode observer-based robust fault reconstruction for a helicopter with actuator fault', Asian Journal of Control 18(4), 1558-1565.

Derbel, S., Feki, N., Barbot, J. P., Nicolau, F., Abbes, M. S. \& Haddar, M. (2019), Electro-mechanical system control based on observers, in 'Advances in Acoustics and Vibration II', Springer International Publishing, pp. 101-110.

Edwards, C., Spurgeon, S. K. \& Patton, R. J. (2000), 'Sliding mode observers for fault detection and isolation', Automatica 36(4), 541-553.

Er-raoudi, M., Diany, M., Aissaoui, H. \& Mabrouki, M. (2016), 'Gear fault detection using artificial neural networks with discrete wavelet transform and principal component analysis', J. Mech. Eng. Sci 10, 2016-2029.

Feki, N., Clerc, G. \& Velex, P. (2010), Modeling of gear-motor dynamic interactions - Applications to the detection of tooth faults by electric measurements, in 'International Conference on Gears', pp. 941-954.

Feki, N., Clerc, G. \& Velex, P. (2013), 'Gear and motor fault modeling and detection based on motor current analysis', Electric Power Systems Research 95, $28-37$.

Foo, G. H. B., Zhang, X. \& Vilathgamuwa, D. M. (2013), 'A sensor fault detection and isolation method in interior permanent-magnet synchronous motor drives based on an extended kalman filter', IEEE Transactions on Industrial Electronics 60(8), 3485-3495.

Galant, A., Beitelschmidt, M. \& Großmann, K. (2016), 'Fast high-resolution fe-based simulation of thermo-elastic behaviour of machine tool structures', Procedia CIRP 46, 627-630. 
Gao, Z., Cecati, C. \& Ding, S. X. (2015), 'A survey of fault diagnosis and faulttolerant techniques - part i: Fault diagnosis with model-based and signal-based approaches', IEEE Transactions on Industrial Electronics 62(6), 3757-3767.

Gao, Z., Liu, X. \& Chen, M. Z. (2015), 'Unknown input observer-based robust fault estimation for systems corrupted by partially decoupled disturbances', IEEE Transactions on Industrial Electronics 63(4), 2537-2547.

Gayatri, C. v. h. \& Sekhar, A. S. (2018), 'Dynamic analysis and characterization of two stage epicyclic gear box in wind turbine drive train', AIP Conference Proceedings 2044(1), 020018.

Gong, X. \& Qiao, W. (2015), 'Current-based mechanical fault detection for direct-drive wind turbines via synchronous sampling and impulse detection', IEEE Transactions on Industrial Electronics 62(3), 1693-1702.

Han, W., Wang, Z. \& Shen, Y. (2018), 'Fault estimation for a quadrotor unmanned aerial vehicle by integrating the parity space approach with recursive least squares', Proceedings of the Institution of Mechanical Engineers, Part G: Journal of Aerospace Engineering 232(4), 783-796.

Hong, L. \& Dhupia, J. S. (2014), 'A time domain approach to diagnose gearbox fault based on measured vibration signals', Journal of Sound and Vibration 333(7), 2164-2180.

Hwang, I., Kim, S., Kim, Y. \& Seah, C. E. (2010), 'A survey of fault detection, isolation, and reconfiguration methods', IEEE Transactions on Control Systems Technology 18(3), 636-653.

Hwang, W. \& Huh, K. (2015), 'Fault detection and estimation for electromechanical brake systems using parity space approach', Journal of Dynamic Systems, Measurement, and Control 137(1), 014504.

Jan, S. U., Lee, Y.-D., Shin, J. \& Koo, I. (2017), 'Sensor fault classification based on support vector machine and statistical time-domain features', IEEE Access 5, 8682-8690.

Kim, K. H., Lee, J. G. \& Park, C. G. (2009), 'Adaptive two-stage extended kalman filter for a fault-tolerant ins-gps loosely coupled system', IEEE Transactions on Aerospace and Electronic Systems 45(1), 125-137.

Mouzakitis, A. (2013), 'Classification of fault diagnosis methods for control systems', Measurement and Control 46(10), 303-308.

Nateghi, S., Shtessel, Y., Barbot, J.-P., Zheng, G. \& Yu, L. (2018), Cyber-attack reconstruction via sliding mode differentiation and sparse recovery algorithm: Electrical power networks application, in '2018 15th International Workshop on Variable Structure Systems (VSS)', IEEE, pp. 285-290.

Odofin, S., Bentley, E. \& Aikhuele, D. (2018), 'Robust fault estimation for wind turbine energy via hybrid systems', Renewable energy 120, 289-299.

Odofin, S., Gao, Z., Liu, X. \& Sun, K. (2016), Robust actuator fault detection for an induction motor via genetic-algorithm optimisation, in '2016 IEEE 11th Conference on Industrial Electronics and Applications (ICIEA)', IEEE, pp. $468-473$.

Rahme, S. \& Meskin, N. (2015), 'Adaptive sliding mode observer for sensor fault diagnosis of an industrial gas turbine', Control Engineering Practice 38, 57-74. 
Rodrigues, M., Hamdi, H., Theilliol, D., Mechmeche, C. \& BenHadj Braiek, N. (2015), 'Actuator fault estimation based adaptive polytopic observer for a class of lpv descriptor systems', International Journal of Robust and Nonlinear Control 25(5), 673-688.

Sen, S. \& Bhattacharya, B. (2017), 'A non-iterative structural damage identification methodology using state space eigenstructure assignment', Structure and Infrastructure Engineering 13(2), 211-222.

Sepasi, M. \& Sassani, F. (2010), 'On-line fault diagnosis of hydraulic systems using unscented kalman filter', International Journal of Control, Automation and Systems 8(1), 149-156.

Sonavane, S. R. \& Jadhav, H. T. (2015), Evolution of wind energy generation system using dfig and fft analysis, in '2015 IEEE 9th International Conference on Intelligent Systems and Control (ISCO)', pp. 1-5.

Tian, J., Morillo, C., Azarian, M. H. \& Pecht, M. (2015), 'Motor bearing fault detection using spectral kurtosis-based feature extraction coupled with knearest neighbor distance analysis', IEEE Transactions on Industrial Electronics 63(3), 1793-1803.

Xu, L. \& Tseng, H. E. (2007), 'Robust model-based fault detection for a roll stability control system', IEEE Transactions on Control Systems Technology 15(3), 519-528.

Youssef, T., Chadli, M., Karimi, H. R. \& Wang, R. (2017), 'Actuator and sensor faults estimation based on proportional integral observer for ts fuzzy model', Journal of the Franklin Institute 354(6), 2524-2542.

$\mathrm{Yu}, \mathrm{Y}$., Woradechjumroen, D. \& Yu, D. (2014), 'A review of fault detection and diagnosis methodologies on air-handling units', Energy and Buildings 82, 550562 . 